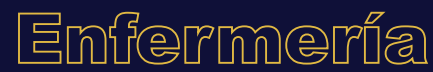

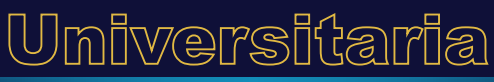

\section{Revisiones Sistemáticas Exploratorias como metodología para la síntesis del conocimiento científico}

\section{Scoping Reviews as a methodology for scientific knowledge synthesis}

\section{Revisões Exploratórias Sistemáticas como metodologia para a síntese do conhecimento científico}

H. Fernández-Sánchez ${ }^{\mathrm{a1}^{*}}$, K. King ${ }^{\mathrm{b} 1}$, C.B. Enriquez-Hernández ${ }^{\mathrm{c2}}$ ORCID:

${ }^{\text {a }}$ https://orcid.org/0ooo-0003-4992-7096

${ }^{\mathrm{b}}$ https://orcid.org/0000-0001-8367-1210

c https://orcid.org/ooo-0oo2-7346-3714

${ }^{1}$ University of Alberta, Faculty of Nursing, Edmonton Clinic Health Academy, Edmonton, Alberta, Canada ${ }^{2}$ Universidad Veracruzana, Facultad de Enfermería, Puerto de Veracruz, Veracruz, México.

Recibido: 26 junio 2019 / Aceptado: 12 diciembre 2019

RESUMEN

Introducción: En la actualidad los sistemas de salud alrededor del mundo apuestan por una toma decisiones clínicas basadas en la evidencia científica. Para ello, es necesario que los profesionales de la salud consulten los resultados de las investigaciones científicas. Sin embargo, dada la gran cantidad de literatura, los investigadores han desarrollado metodologías de revisión para compilar los estudios científicos dentro de un área específica. Aun cuando existen más de 10 tipos de metodologías para la revisión de la literatura, la Revisión Sistemática Exploratoria (RSE) ha recibido poca atención en la literatura sobre métodos de investigación científica de habla hispana.

Objetivo: Detallar la metodología de la RSE, sus propósitos y las fases para su desarrollo. Desarrollo: Este trabajo detalla las generalidades de la RSE basándose en la metodología propuesta por Arksey y O’Malley. Así mismo, se describen las áreas o ámbitos donde este tipo de revisión se puede emplear, las fases para desarrollar la revisión y ejemplos de las RSE. Conclusiones: Las RSE tienen la fortaleza de hacer saber a los profesionales de la salud sobre un tema en específico que permita incidir en las políticas públicas. Al igual que las Revisiones

*Autor para correspondencia. Correo electrónico: higinio@ualberta.ca https://doi.org/10.22201/eneo.23958421e.2020.1.697

1665-7063/@ 2020 Universidad Nacional Autónoma de México, Escuela Nacional de Enfermería y Obstetricia. Este es un artículo Open Access bajo la licencia CC BY-NC-ND (http://creativecommons.org/licenses/by-nc-nd/4.o/). 
Sistemáticas, las RSE utilizan una metodología clara y replicable, aportando datos confiables y científicos para los profesionales de la salud.

Palabras clave: Método; revisión sistemática; revisión sistemática exploratoria; Canadá.

\section{INTRODUCCIÓN}

El nacimiento de la Practica Basada en Evidencia (PBE) ha dado pauta a la toma de decisiones clínicas basadas en la evidencia científica' ${ }^{1}$. Sin embargo, dada la gran cantidad de literatura existente, resulta

Keywords: Method; scoping review; systematic review; Canada.

\section{RESUMO}

Introdução: Atualmente, os sistemas de saúde em todo o mundo apostam em tomar decisões clínicas baseadas em evidências científicas. Para isso, é necessário que os profissionais de saúde consultem os resultados da pesquisa científica. No entanto, dada a grande quantidade de literatura, os pesquisadores desenvolveram metodologias de revisão para compilar estudos científicos dentro de uma área específica. Embora existam mais de 10 tipos de metodologias para a revisão da literatura, a Revisão Explorada Sistemática (ESR) recebeu pouca atenção na literatura sobre métodos de pesquisa científica de língua espanhola.

Objetivo: Detalhar a metodologia da RSC, seus propósitos e as fases para o seu desenvolvimento.

Desenvolvimento: Este artigo detalha as generalidades da RSC com base na metodologia proposta por Arksey e O’Malley (2005). Ele também descreve as áreas ou áreas onde esse tipo de revisão pode ser usado, as fases para desenvolver a revisão e exemplos de RSC.

Conclusões: As RSCs têm a força para permitir que os profissionais de saúde conheçam uma questão específica que permite influenciar as políticas públicas. Como as Revisões Sistemáticas, a RSE usa uma metodologia clara e replicável, fornecendo dados confiáveis e científicos para profissionais de saúde.

Palavras-chave: Método; revisão sistemática; revisão sistemática exploratória; Canada.

\begin{abstract}
Introduction: Currently, health systems around the world are betting on making clinical decisions based on scientific evidence. For this, it is essential that healthcare providers consult the results of scientific research. However, given the large amount of literature, researchers have developed knowledge synthesis methodologies to compile scientific studies within a specific area. Although there are more than 10 types of methodologies for reviewing the literature, Scoping Reviews have received little attention in the literature on Spanish-speaking scientific research methods.

Objective: Detail the Scoping Review methodology, its purposes and the phases for its development.

Development: This paper details the generalities of Scoping Reviews based on the methodology proposed by Arksey \& O'Malley. It also describes the areas where this type of review can be employed, the phases to develop the review and examples.

Conclusions: Scoping Reviews have the strength to inform healthcare providers and policy on a specific topic of interest. Like Systematic Reviews, Scoping Reviews uses a clear and replicable methodology, providing reliable and scientific data for healthcare providers, policy makers and researchers.
\end{abstract}


difícil para los profesionales de la salud consultar los resultados de las investigaciones ${ }^{2-3}$. Ante este fenómeno, surge la necesidad de crear metodologías para la síntesis de conocimiento científico. En un inicio, surgió la metodología de las revisiones sistemáticas (RS). Las RS se han realizado en gran medida para explorar, evaluar y sintetizar la evidencia científica sobre la efectividad de los ensayos clínicos aleatorizados (ECA $)^{4-5}$. Las revisiones sistemáticas elaboradas bajo los lineamientos de la Colaboración Cochrane se consideran como la fuente más confiable de evidencia científica para guiar la práctica clínica4-5. No obstante, las RS se han realizado en mayor medida para compilar la evidencia científica de los ECA. Este fenómeno ha permitido la creación de otro tipo de revisiones, que permiten resumir los resultados de diversas fuentes de evidencia científica (i.e. estudios de caso, estudios cualitativos, estudios teóricos, literatura gris ${ }^{6-8}$. Por un lado, Grant y Booth ${ }^{9}$ identificaron 14 tipos de revisiones; revisión crítica, revisión de literatura, revisión de mapeo, revisión de métodos mixtos, revisión descriptiva, revisión sistemática cualitativa, revisión rápida, metaanálisis, revisión del estado del arte, revisión sistemática, revisión sistematizada, revisión paraguas, revisión de búsqueda y revisión sistemática exploratoria. Esta última, permite abordar preguntas amplias que proporcionan una visión general de la evidencia científica disponible. Principalmente, las revisiones sistemáticas exploratorias (RSE) plantean y abordan preguntas que permiten identificar vacíos en la base de evidencia donde no se cuenta con ninguna investigación y cuando aún no es claro si una revisión sistemática es necesaria para áreas específicas de la investigación?. La metodología de las RSE nace en el año $2005^{10}$, a partir de la publicación, se ha avanzado en su sistematización, 2010 211 y $2017^{8}$. La profundidad en la sistematización de la metodología ha permitido que los resultados generados de las RSE puedan orientar la práctica, las políticas y la investigación.

Ante este panorama y con la finalidad de ampliar el uso de las revisiones sistemáticas exploratorias (RSE) en el ámbito de la investigación científica, este trabajo tiene como objetivo detallar la metodología de la Revisión Sistemática Exploratoria, sus propósitos y las fases para su desarrollo.

\section{DESARROLLO}

\section{Generalidades de la RSE}

Arksey y O'Malley ${ }^{10}$ hicieron la primera publicación formal de la metodología para la RSE, está consiste en cinco fases. Años más tarde, Levac, Colquhoun y O'Brien ${ }^{11}$ se dieron a la tarea de proporcionar una explicación más detallada de cada una de las fases de esta metodología; esto permitió aumentar la claridad y el rigor del proceso de revisión. En 2015, el Instituto de Joanna Briggs ${ }^{12}$ amplió el proceso de la revisión y aumentó cuatro fases más; como resultado, la RSE quedó en nueve fases. Al mismo tiempo, el grupo PRISMA publicó una extensión de su diagrama de flujo para mostrar el proceso de selección de artículos para las RSE, la cual se deriva de los elementos para las Revisiones Sistemáticas y los Metanálisis ${ }^{12}$.

\section{Propósitos de la RSE}

Las RSE se pueden realizar para dar respuestas a una serie de preguntas de investigación; a) examinar el alcance, rango y naturaleza de la evidencia científica existente; b) determinar si una Revisión Sistemática sería necesaria para el fenómeno de interés; c) resumir y difundir los hallazgos de la evidencia existente; d) identificar los vacíos del conocimiento en la evidencia científica existente; e) aclarar conceptos clave e informar sobre los tipos de estudios que abordan e informan la práctica clínica en una área en particular; f) mapear la evidencia científica existente y/o documentos de políticas públicas e informes que guían la práctica clínica en un área en particular ${ }^{8-10}$. 


\section{Fases de la Metodología de la RSE}

A pesar de que el Instituto de Joanna Briggs tiene un capitulo completo de la metodología para $\mathrm{RSE}^{8}$, la metodología propuesta por Arksey y O’Malley ${ }^{10}$ continúa siendo la más utilizada. Por ende, a continuación, describimos las cinco fases para desarrollar una Revisión Sistemática Exploratoria de acuerdo con Arksey y O'Malley ${ }^{10}$ y Levac, Colquhoun y O'Brien ${ }^{11}$. Dichas fases consisten en: a) identificación de la pregunta de investigación; b) búsqueda sistematizada de la evidencia científica; c) selección de los estudios; d) extracción de los datos; y e) recopilación, resumen y difusión de los resultados ${ }^{10}$ (Tabla 1).

Tabla 1. Fases de las RSE de acuerdo con Arksey y O'Malley

\begin{tabular}{|c|c|}
\hline Fases & Resumen \\
\hline $\begin{array}{l}\text { Fase I } \\
\text { Elaboración de la } \\
\text { pregunta. }\end{array}$ & $\begin{array}{l}\text { a. Elaborar la pregunta de investigación en relación con el/los objetivo(s) de la RSE. } \\
\text { b. Detallar el cuerpo de literatura que se va a resumir y para quién se está } \\
\text { resumiendo. }\end{array}$ \\
\hline $\begin{array}{l}\text { Fase II } \\
\text { Establecimiento de los } \\
\text { criterios de inclusión y } \\
\text { exclusión y búsqueda } \\
\text { sistemática. }\end{array}$ & $\begin{array}{l}\text { a. Establecer los criterios de inclusión (i.e. años, idiomas, tipo de evidencia). } \\
\text { b. Seleccionar las palabras clave y los términos de búsqueda. Consultar las paginas } \\
\text { MeSH y/o DeCS. } \\
\text { c. Elaborar una estrategia de búsqueda para cada fuente de información. Consultar } \\
\text { con una bibliotecaria. } \\
\text { d. Describir todas las fuentes de información en la búsqueda (i.e. bases de datos } \\
\text { electrónicas). }\end{array}$ \\
\hline $\begin{array}{l}\text { Fase III } \\
\text { Revisión y selección } \\
\text { de estudios. }\end{array}$ & $\begin{array}{l}\text { a. Identificar y eliminar estudios que sean duplicados. } \\
\text { b. Revisar títulos y resúmenes. Dos revisores de manera independientes realizan } \\
\text { este paso utilizando los criterios de inclusión y exclusión. } \\
\text { c. De los artículos restantes se hace la lectura del artículo completo. Dos revisores } \\
\text { de manera independiente realizan este paso utilizando los criterios de inclusión } \\
\text { y exclusión. } \\
\text { d. De los artículos que se incluirán en el análisis, se hace una búsqueda de las listas } \\
\text { de referencias para detectar estudios relevantes que no fueron capturados en la } \\
\text { búsqueda en las bases de datos. } \\
\text { *Para este paso es ideal que se utilicen programas como RefWorks, EndNote, } \\
\text { Covidence, etc. }\end{array}$ \\
\hline $\begin{array}{l}\text { Fase IV } \\
\text { Extracción de datos. }\end{array}$ & $\begin{array}{l}\text { e. Se realiza la extracción de los datos de acuerdo con las necesidades de cada RSE } \\
\text { (i.e. objetivo, diseño, muestra, contexto, resultados relevantes). } \\
\text { f. La extracción la puede realizar un revisor y un segundo la confirma; o bien, dos } \\
\text { revisores extraen los datos y posteriormente la comparan. }\end{array}$ \\
\hline $\begin{array}{l}\text { Fase } \mathrm{V} \\
\text { Análisis y reporte de } \\
\text { los resultados. }\end{array}$ & $\begin{array}{l}\text { a. Los resultados de estudios cuantitativos se analizan de manera numérica, } \\
\text { mientras los hallazgos de los estudios cualitativos se analizan utilizando el } \\
\text { análisis temático. }\end{array}$ \\
\hline
\end{tabular}

La Primera Fase consiste en elaborar la pregunta de investigación. La pregunta de investigación debe estar claramente estructurada, de tal modo que se pueda establecer una estrategia de búsqueda sistemática efectiva. Además, para visualizar el resultado esperado se deben considerar conjuntamente el propósito y la pregunta de investigación de las RSE. Uno de los formatos más utilizados para formular las preguntas de las RSE es el formato CPC (Concepto, Población y Contexto) $)^{8}$. Sin embargo, también se han utilizado el formato PICO (Persona/Población, Intervención, Comparación y Resultado) ${ }^{13}$ y el formato SPIDER (Muestra, Fenómeno de Interés, Diseño, Evaluación y Tipo de Investigación) $)^{13}$. Un ejemplo de pregunta aplicando el formato CPC es: ¿Cuál es el alcance y la naturaleza de la literatura existente sobre la violencia (concepto) contra las enfermeras/os (población) en el lugar de trabajo (contexto)? Está pregunta de investigación es apropiada para las RSE, ya que no se especifica el tipo de violencia (i.e. laboral, sexual, psicológica), ni características 
específicas de la población (i.e. nivel de estudios, servicio, antigüedad) y tampoco se limita al tipo de literatura (i.e. literatura gris, científica).

La Segunda Fase consiste en predeterminar los criterios de inclusión y exclusión. Estos deben estar alineados con la pregunta de investigación y con los objetivos ${ }^{8,10-11}$. Estos pueden incluir fechas, idiomas, tipo de fuentes de información (i.e. artículos científicos publicados, literatura gris). Además, se establecen los términos que se utilizarán para realizar la búsqueda en las bases de datos (i.e. concepto, población, contexto). En lo que respecta a ciencias de la salud, los conceptos pueden ser verificados en la página Medical Subject Headings (MeSH) o en la página Descriptores en Ciencias de la Salud (DeCS). Estas páginas proporcionan terminología estructurada que permite la búsqueda y recuperación de artículos en revistas científicas en las bases de datos electrónicas (i.e. MedLine, CINAHL). Enseguida, se realiza una búsqueda exhaustiva y sistemática de la literatura en las bases de datos más populares en las áreas de interés (i.e. ciencias de la salud, ciencias sociales y género). La pesquisa consiste en desarrollar estrategias de búsqueda aplicando los operadores booleanos (AND, OR, NOT). Dado que cada base de datos electrónica mantiene una búsqueda particular, es recomendable consultar con una bibliotecaria y así obtener resultados concretos e información de mejor calidad.

La Tercera Fase se desarrolla en tres pasos. Primero, se detectan y se excluyen duplicados de estudios. Es posible que un mismo artículo se encuentre archivado en más de una base de datos, es por ello la importancia de detectar dichos duplicados. En la actualidad, los equipos de investigación utilizan gestores de referencias bibliográficas (i.e. Refworks, EndNote) u otro software (i.e. Covidence) que facilitan el proceso de detección de duplicados. En el segundo, dos revisores independientes analizan los títulos y resúmenes de los artículos restantes, esto permite analizar de manera rápida si el estudio cumple con los criterios de inclusión. En el tercero, se hace la lectura completa de los artículos incluidos por dos revisores independientes. La inclusión y/o exclusión de los estudios dependerá de los criterios predeterminados por el equipo de investigación. Los conflictos que surjan entre revisores pueden ser resueltos por consenso (entre revisores) o bien por un tercer revisor (otro miembro del equipo de investigación) ${ }^{10-11}$. Por último, una vez que se tenga el número final de estudios que se incluirán para el análisis, se recomienda realizar una revisión de la lista de referencias de dichos artículos. Esto permite la identificación de manera manual de estudios adicionales, que no fueron capturados en la búsqueda sistematizada en las bases de datos electrónicas.

Al igual que en el paso uno, los revisores pueden recurrir a la tecnología para ejecutar los pasos dos y tres. Una de las herramientas tecnológicas utilizadas con mayor frecuencia es Covidence, un software basado en la web que organiza revisiones de síntesis del conocimiento ${ }^{14}$. Este software funciona en colaboración con la Biblioteca Cochrane para optimizar el proceso de revisión. Covidence permite: a) identificar duplicados; b) realizar procesos independientes; c) resolución de conflictos entre revisores; d) almacena artículos de texto completo; y e) permite exportar datos (i.e. Excel).

En la Fase Cuatro, los revisores hacen la extracción de datos de los artículos incluidos para el análisis final. Este apartado se puede realizar de dos formas: a) un revisor extrae los datos y otro revisa y verifica la información; o bien, b) dos revisores extraen los datos de manera independiente para después comparar la información. El Instituto de Joanna Briggs ${ }^{8}$ recomienda utilizar una guía o formato de extracción de datos diseñados para las RSE; sin embargo, éste puede ser adaptado para que se ajuste a las necesidades de cada revisión.

Finalmente, en la Fase Cinco, los resultados se resumen, se cotejan y se reportan ${ }^{10-11}$. Para ello, los hallazgos se sintetizan empleando la estadística descriptiva (numérica) para los datos cuantitativos 
y un análisis temático para los datos cualitativos. Además, en esta fase los autores deben identificar las implicaciones que los hallazgos tienen sobre las políticas públicas y de salud, la práctica y/o la investigación científica. No obstante, antes de iniciar con la redacción del manuscrito para su publicación es recomendable que se vuelva a realizar la búsqueda en las bases de datos electrónicas para capturar estudios publicados recientemente. Ésta es considerada una buena práctica, ya que el tiempo aproximado en realizar una RSE es de seis a 12 meses o más.

\section{Ejemplos de Publicaciones con la Metodología de la RSE}

Investigadores y académicos de diversas disciplinas han utilizado la metodología de las RSE para sus trabajos científicos en diversas áreas de la salud (i.e. migración, adicciones, enfermedades crónico-degenerativas, salud sexual y reproductiva). Salami, Meharali y Salami ${ }^{15}$ publicaron una RSE sobre la salud de los trabajadores temporales en Canadá; O'Rourke et al. ${ }^{16}$ investigaron la definición, los determinantes y resultados de la conexión social de los adultos mayores, y Jang, Johnson, D'EramoMelkus y Vordersrasse ${ }^{17}$ exploraron la participación de minorías raciales y étnicas en intervenciones basadas en la tecnología para el autocuidado de la diabetes tipo 2. Investigadores también han acudido a las RSE para diseñar trabajos de intervención en diversas áreas: salud mental ${ }^{18-19}$, rehabilitación física ${ }^{20-21}$, sobrepeso y obesidad ${ }^{22}$, adicciones ${ }^{23}$, salud laboral ${ }^{24}$, cuidados intensivos ${ }^{25}$.

\section{CONCLUSIONES}

Sin lugar a dudas las Revisiones Sistemáticas Exploratorias son una herramienta fundamental para investigadores, académicos y personal clínico. Las RSE comparten varias características con las Revisiones Sistemáticas en el intento de ser sistemático, transparente y replicable. Además, permite informar sobre las políticas públicas en salud y a los profesionales de la salud. No obstante, la evaluación de calidad de los estudios es opcional; por lo tanto, la mayoría de las RSE no la realizan. Esta limitación metodológica puede resultar en riesgo de sesgo por parte de los investigadores. En consecuencia, los hallazgos no pueden ser utilizados para recomendar políticas en salud y/o cambios en la práctica clínica.

\section{RESPONSABILIDADES ÉTICAS}

Protección de personas y animales. No procede, ya que en esta investigación se trabajó únicamente con documentos.

Conflicto de intereses. Los autores declaran no tener conflicto de intereses.

Financiamiento. Este trabajo fue financiado por donadores del Hospital Lois Hole para Mujeres a través del Instituto de Investigación de Salud de Mujeres y Niños (Women and Children's Health Research Institute [WCHRI]). Así mismo, por la beca de estudios en el extranjero a través del Consejo Nacional de Ciencia y Tecnología (CONACyT).

\section{REFERENCIAS}

1. Mazurek-Melnyk B, Fineout-Overholt E, Stillwell SB, Williamson KM. The seven steps of evidencebased practice: following this progressive, sequential approach will lead to improved health care and patient outcomes. Am J Nurs. 2010; 11O(1): 51-3. https://doi.org/10.1097/01.NAJ.0000366056.06605.d2

2. Wachtel RE, Dexter F. Difficulties and challenges associated with literature searches in operating room management, complete with recommendations. Anesth Analg. 2013; 117(6): 1460-79. https://doi.org/10.1213/ANE.obo13e3182a6d33b. 
3. Wallace J, Nwosu B, Clarke M. Barriers to the uptake of evidence from systematic reviews and meta-analyses: a systematic review of decision makers' perceptions. BMJ Open. 2012; 2(5): eoo122O. http://dx.doi.org/10.1136/bmjopen-2012-001220

4. Clarke J. What is a systematic review? Evid Based Nurs. 2011. 14(3): 64. http://dx.doi.org/10.1136/ebn.2011.0049

5. Higgings JPT, Green S (editors). Cochrane handbook for systematic reviews of interventions Version 5.1.o. The Cochrane Collaboration; 2011. http://bit.ly/2S2zCru

6. Schick-Makaroff K, MacDonald M, Plummer M, Burgess J, Neander W. What synthesis methodology should I use? A review and analysis of approaches to research synthesis. AIMS Public Health. 2016; 3(1): 172-215. https://doi.org/10.3934/publichealth.2016.1.172

7. Paré G, Trudel MC, Jaana M, Kitsiou S. Synthesizing information systems knowledge: A typology of literature reviews. Inform Manage-Amster. 2015; 52(2):183-99. https://doi.org/10.1016/j.im.2014.08.008

8. Peters MDJ, Godfrey C, McInerney P, Baldini-Soares C, Khalil H, Parker D. Chapter 11: Scoping Reviews. En: Aromataris E, Munn Z (Editors). Joanna Briggs Institute Reviewer's Manual. The Joanna Briggs Institute; 2017. https://bit.ly/2txKdkO

9. Grant $M J$, Booth A. A typology of reviews: an analysis of 14 review types and associated methodologies. Health Info Libr J. 2009; 26(2): 91-108. https://doi.org/10.1111/j.1471-1842.2009.00848.x

10. Arksey H, O'Malley L. Scoping studies: towards a methodological framework. Int J Soc Res Methodol. 2005; 8(1): 19-32. https://doi.org/10.1080/1364557032000119616

11. Levac D, Colquhoun H, O'Brien KK. Scoping studies: advancing the methodology. Implement Sci. 2010; 5 (Article number 69): 9 screens. https://doi.org/10.1186/1748-5908-5-69

12. Tricco AC, Lillie E, Zarin W, O'Brien KK, Colquhoun H, Levac D, et al. PRISMA extension for scoping reviews (PRISMA-ScR): checklist and explanation. Ann Intern Med. 2018; 169(7): 467-73. https://doi.org/10.7326/M18-0850

13. Cooke A, Smith D, Booth A. Beyond PICO: The SPIDER tool for qualitative evidence synthesis. Qual Health Res. 2012; 22(10): 1435-43. https://doi.org/10.1177/1049732312452938

14. Cochrane Library. About Covidence. Community.cochrane.org. United Kingdom: Cochrane comunnity; 2020. https://bit.ly/2OvQ_oLO

15. Salami B, Meharali S, Salami A. The health of temporary foreign workers in Canada. Can J Public Health. 2015; 106(8): 546-54. https://doi.org/10.17269/cjph.106.5182.

16. O’Rourke HM, Sidani S, Chu CH, Fox M, McGilton KS, Collins J. Pilot of a tailored danceiIntervention to support function in people with cognitive impairment residing in long-term care: A brief report. Gero and Geriatric Med. 2017; 3: 1-8. https://doi.org/10.1177/2333721417734672

17. Jang M, Johnson CM, D'Eramo-Melkus G, Vorderstrasse AA. Participation of racial and ethnic minorities in technology-based interventions to self-manage type 2 diabetes: A scoping review. J Transcult Nurs. 2018; 29(3):292-307. https://doi.org/10.1177/1043659617723074

18. Fernández-Sánchez H, Enríquez-Hernández CB, Sidani S, Hernández-Osorio C, Castellanos-Contreras E, Salazar-Mendoza J.Dance intervention for Mexican family caregivers of children with developmental disability: A pilot study. J Transcult Nurs. 2019; 31(1):38-44. https://doi.org/10.1177/1043659619838027

19. O'Rourke HM, Collins L, Sidani S. Interventions to address social connectedness and loneliness for older adults: a scoping review. BMC geriatr. 2018; 18(1): 214. https://doi.org/10.1186/s12877-018-0897-x

20. Reid JC, Unger J, McCaskell D, Childerhose L, Zorko DJ, Kho ME. Physical rehabilitation interventions in the intensive care unit: a scoping review of 117 studies. J intensive care. 2018; 6(1):80.

https://doi.org/10.1186/s4056o-018-0349-x 
21. McArthur C, Gibbs JC, Patel R, Papaioannou A, Neves P, Killingbeck J, et al. A scoping review of physical rehabilitation in long-term care: Interventions, outcomes, tools. Ca J Aging. 2017; 36(4):435-52. http://dx.doi.org/10.1017/So71498081700040X

22. Bussiek P-BV, De Poli C, Bevan G. A scoping review protocol to map the evidence on interventions to prevent overweight and obesity in children. BMJ Open 2018; 8(2): eo19311. http://dx.doi.org/10.1136/bmjopen-2017-019311.

23. Gardner K, Kearns R, Woodland L, Silveira M, Hua M, Katz M, et al. A scoping review of the evidence on health promotion interventions for reducing waterpipe smoking: implications for practice. Front Public Health. 2018; 6: article 308. https://doi.org/10.3389/fpubh.2018.00308

24. Verbeek J, Ruotsalainen J, Laitinen J, Korkiakangas E, Lusa S, Mänttäri S, et al. Interventions to enhance recovery in healthy workers; a scoping review. Occup Med. 2019; 69(1):54-63. https://doi.org/10.1093/occmed/kqy141.

25. Hammond DA, Gurnani PK, Flannery AH, Smetana KS, Westrick JC, Lat I, et al. Scoping review of interventions associated with cost avoidance able to be performed in the intensive care unit and emergency department. Pharmacotherapy. 2019; 39(3):215-31. https://doi.org/10.1002/phar.2224 